\title{
Where Have All the Medicare Inpatients Gone?
}

\author{
Ifedayo O Kuye, MD, MBA ${ }^{1 *}$, Scott A Berkowitz, MD, MBA ${ }^{2}$
}

${ }^{1}$ Division of Hospital Medicine, Department of Medicine, Johns Hopkins University School of Medicine, Baltimore, Maryland; ${ }^{2}$ Division of Cardiology, Department of Medicine, Johns Hopkins University School of Medicine, Baltimore, Maryland.

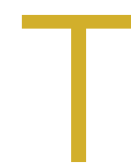

he advent of COVID-19 saw a precipitous decline in inpatient admissions. Even before the COVID-19 pandemic, hospitals were seeing a trend toward fewer inpatient admissions for Medicare beneficiaries, which has not been thoroughly examined or explained. ${ }^{1}$ In this issue, Keohane et al ${ }^{2}$ studied Medicare inpatient episode trends between 2009 and 2017 and found that, during this period, inpatient episodes per 1000 Medicare fee-for-service (FFS) beneficiaries declined by $18.2 \%$, from 326 to 267 per 1000 beneficiaries.

This trend can be partly explained by changes in the way that care is delivered. First, observation stays have risen, and these are excluded in the authors' analysis. From 2010 to 2017, observation visits per 1000 beneficiaries increased from 28 to $51 .{ }^{1}$ Second, due to improved outpatient management, margin constraints, and efficiency gains, hospitals are less likely to admit patients with less complex problems or keep patients overnight for uncomplicated procedural interventions. In cardiology, there has been an increase in the proportion of same-day percutaneous coronary interventions, from $4.5 \%$ in 2009 to $28.6 \%$ in $2017 .{ }^{3}$ The authors do not include a quantitative measure of complexity, but their data support this conclusion as they find larger declines in episodes that began with a planned admission and those that involved no use of post-acute care services, and thus were likely less complicated admissions. Finally, the increased use of alternative care sites such as home-based care settings and urgent care clinics, the proliferation of telemedicine, and the continual development of guideline-based therapy have resulted in better outpatient management of diseases.

The growth of value-based care has also contributed to the reduction in inpatient admission. The past decade has seen the growth of bundled-payment contracts, accountable care organizations (ACO), and advanced primary care models. In 2018, an estimated $20 \%$ of Medicare beneficiaries were part of an $\mathrm{ACO}{ }^{4}$ These changes have led healthcare systems to invest in care management and postdischarge interventions, such as postdischarge phone calls, transitional clinics, and transition guides to reduce admissions and readmissions. Johns Hopkins adopted all these strategies to drive performance on the Maryland Total Cost of Care Model, which like an ACO holds hospitals accountable for both inpatient and outpatient costs incurred by Medicare FFS beneficiaries. A consistent theme among successful ACOs has been a reduction in inpatient spending. ${ }^{5}$

The authors are likely undercounting the volume of admissions by Medicare beneficiaries. First, to define an episode, they leverage the Medicare definition of bundles and include traditional Medicare inpatient, outpatient, and Part D services

*Corresponding Author: Ifedayo O Kuye, MD, MBA;

Email: ikuye1@jhmi.edu; Telephone: 410-955-5000.

Received: October 21, 2021; Accepted: October 21, 2021

๑) 2021 Society of Hospital Medicine DOI 10.12788/jhm.3725
30 days prior to hospitalizations and up to 90 days after. Admissions for the same diagnosis related group that occur in the 90 days after the anchor hospitalization are included in the same episode. From a clinical perspective, it is not intuitively clear why an admission for heart failure or pneumonia that occurs 3 months after an anchor hospitalization would not be defined as a separate and distinct admission rather than a readmission. Second, their analysis focuses on Medicare FFS and does not include Medicare Advantage, which now accounts for $42 \%$ of total Medicare beneficiaries. In fact, Medicare Advantage experienced significant growth in enrollment during the study period, increasing from 10 million to 24 million beneficiaries. ${ }^{6}$

Despite the reduction in inpatient volumes, the authors find that inpatient spending has increased. Spending per episode increased by $11.4 \%$ over this period, when adjusted for Medicare payment increases. Actual spending per episode unadjusted for payment increases rose by $25 \%$. Thus, they astutely point out that most of the increase has been driven by Medicare payment increases. It is likely that increases in the complexity of patients and more dedicated focus on appropriate coding have also contributed. The authors, however, do not provide information on changes to the total cost of care outside of their defined inpatient episodes, a relevant measure to those participating in value-based models.

It is likely that the trend toward fewer inpatient admissions and increased outpatient management of medical conditions will continue as value-based care models grow. Studies like these are important in documenting this trend, but it will be important in future studies to understand how these changes have impacted the quality of care delivered to patients. Prior studies have found that reductions in readmissions through the Hospital Readmission Reduction Program were associated with increases in mortality as a potential unintended consequence?

Disclosures: The authors reported no conflicts of interest.

References

1. The Medicare Payment Advisory Commission. A Data Book: Health Care Spending and the Medicare Program. June 2018. Accessed October 25, 2021. http://medpac. gov/docs/default-source/data-book/jun19_databook_entirereport_sec.pdf

2. Keohane LM, Kripalani S, Buntin MB, et al. Traditional Medicare spending on inpatient episodes as hospitalizations decline. J Hosp Med. 2021;16(11): 652-658. https://doi.org/10.12788//hm.3699

3. Bradley SM, Kaltenbach LA, Xiang K, et al. Trends in use and outcomes of sameday discharge following elective percutaneous coronary intervention. JACC Cardiovasc Interv. 2021;14(15):1655-1666. https://doi.org/10.1016/j.jcin.2021.05.043

4. National Association of ACOs. NAACOs overview of the 2018 Medicare ACO class. Accessed October 25, 2021. https://www.naacos.com/overviewof-the-2018-medicare-aco-class

5. McWilliams JM, Hatfield LA, Landon BE, Hamed P, Chernew ME. Medicare spending after 3 years of the Medicare shared savings program. N Engl J Med. 2018;379(12):1139-1149. https://doi.org/10.1056/NEJMsa1803388

6. Freed M, Fuglesten Biniek J, Damico A, Neuman T. Medicare Advantage in 2021: Enrollment update and key trends. KFF. June 21, 2021. Accessed October 25, 2021. https://www.kff.org/medicare/issue-brief/medicare-advantagein-2021-enrollment-update-and-key-trends/

7. Gupta $A$, Allen LA, Bhatt $D L$, et al. Association of the hospital readmissions reduction program implementation with readmission and mortality outcomes in heart failure. JAMA Cardiol. 2018;3(1):44-53. https://doi.org/10.1001/jamacardio.2017.4265 